East African Medical Journal Vol. 80 No 4 April 2003

ANTIMICROBIAL SUSCEPTIBILITY IN COMMUNITY-ACQUIRED BACTERIAL PNEUMONIA IN ADULTS

S. Kariuki, PhD, J. Muyodi, Dip. Lab. Tech., Centre for Microbiology Research, Kenya Medical Research Institute, P.O. Box 43640, Nairobi, Kenya, B. Mirza, MD, Nairobi Hospital, P.O. Box 30026, Nairobi, W. Mwatu, B. Pharm, GlaxoSmithKIine Pharmaceuticals, P.O. Box 78392, Nairobi, Kenya and J.J.D. Daniels, PhD, GlaxoSmithKline, Africa \& Middle East CDMA, 980 Great West Road, Brentford, Middlesex, United Kingdom

Request for reprints to: Dr. S. Kariuki, Centre for Microbiology Research, Kenya Medical Research Institute, P.O. Box 43640, Nairobi, Kenya

\title{
ANTIMICROBIAL SUSCEPTIBILITY IN COMMUNITY-ACQUIRED BACTERIAL PNEUMONIA IN ADULTS
}

\section{S. KARIUKI, J. MUYODI, B. MIRZA, W. MWATU and J.J.D. DANIELS}

\begin{abstract}
Objectives: To determine the antimicrobial susceptibility patterns of Streptococcus pneumoniae and Haemophilus influenzae, two bacterial pathogens commonly associated with communityacquired pneumonia.

Design: Cross-sectional study.

Setting: Bacterial isolates were obtained from adults suspected to have community-acquired pneumonia and who sought treatment at two city council clinics in Nairobi, Kenya. Susceptibility to antimicrobial agents was performed using a microdilution broth method, according to the criteria set by the National Committee for Clinical Laboratory Standards. Results: A total of 277 S. pneumoniae and $58 \mathrm{H}$. influenzae were obtained from 536 adults examined in the period January 1998 to December 1999. Of the 277 S. pneumoniae, only $56.7 \%$ were susceptible to penicillin and $7.6 \%$ of strains were resistant to two or more antimicrobial agents. Of the $58 \mathrm{H}$. influenzae strains, $91.4 \%$ were sensitive to ampicillin, with $6.8 \%$ resistant to two or more antimicrobial agents. $8.6 \%$ were $B$-lactamase producers and accounted for the entire ampicillin-resistant population.

Conclusion: The prevalence of resistance to penicillin and other commonly used antibiotics among pneumococci is high and the large number of multi-resistant strains among $\boldsymbol{H}$. influenzae is a cause for concern. The prudent use of antibiotics in treatment of pneumonia and other infections should be advocated to minimise spread of resistance.
\end{abstract}

\section{INTRODUCTION}

Respiratory tract infections are the most frequent reason for antibiotic prescription in ambulant patients. In most countries, Streptococcus pneumoniae and Haemophilus influenzae are the major causes of community-acquired pneumonia. Additionally in immunosuppressed patients, Pseudomonas spp, Staphylococcus aureus and Pneumocystis carinii may also be important causes of pneumonia. Indeed in subSaharan Africa, respiratory tract infections are the most common causes of morbidity and mortality in HIV-1 infected adults $(1,2)$. A recent study in hospitalised adults in Kenya with pneumonia (3) found that $S$. pneumoniae was documented in more than half of the patients while $H$. influenzae was the causative agent in $4 \%$ of patients.

Data from surveillance studies in the USA and Canada show that $15-57 \%$ of S. Pneumoniae isolates are intermediately or highly resistant to penicillin (4). Resistance to macloride agents often occurs concurrently with penicillin resistance (5), and these too have been on the rise. Currently in several parts of the world including the UK (6), Indonesia (7), South Africa (8), Brazil (9) and in four North African countries (10), the prevalence of multidrug resistant (MDR) pneumococci (resistant to 2 or more commonly used drugs including penicillin, doxycycline and cotrimoxazole) has been on the increase. However, these strains have remained fairly susceptible to newer quinolones and extended spectrum cephalosporins. In a recent study in Kenya (3) S. pneumoniae isolates from adults were either susceptible (MIC $<1 \mu \mathrm{g} / \mathrm{mL}$ ) or intermediate $(1-2 \mu \mathrm{g} / \mathrm{mL})$ to penicillin. Resistance to erythromycin was $1.5 \%$, to cotrimoxazole $54 \%$, to doxycycline $32 \%$ and chloramphenicol $3 \%$.

Resistance to ß-lactams in Haemophilus influenzae is almost exclusively due to production of TEM-1 $\beta$ lactamases (95\%) and ROB-1 enzymes (5\%), both enzymes are plasmid-mediated. In $66.7 \%$ of recent $H$. influenzae isolates from USA and Canada the presence of ROB-1 correlated with cefaclor resistance that may be the result of mutations or change in expression in the ROB-1 Blactamase gene (11). In the USA since 1983, the prevalence of B-lactamase producing $H$. influenzae has continued to rise from $14.5 \%$ to $37.5 \%$ in 1997 , providing resistance to 
penicillin and first generation cephalosporins (5). However, strains remained susceptible to extended spectrum cephalosporins.

Isolation, identification and susceptibility testing to guide treatment is not feasible for each individual patient because of the time required for the laboratory procedures. Treatment is therefore usually empirical. Empirical treatment should be based on the knowledge of local susceptibility levels of the most likely pathogens. These patterns must, therefore, be continually monitored, as resistance among both community and hospital acquired pathogens is increasing. The present study presents surveillance data on the antibacterial susceptibility of the key respiratory pathogens $S$. pneumoniaeand $H$. influenzae.

\section{MATERIALS AND METHODS}

Patients: These were adults who sought care at the outpatient clinics at Mbagathi District Hospital and the Nairobi City Council Chest Clinic, and were treated for acute respiratory infections. Patients were recruited for a period of two years, January 1998 to December 1999. Specimens including sputum, and throat swabs were obtained from each case before treatment was commenced. In addition S. pneumoniae isolates were obtained from bronchoalveolar lavage and blood cultures from a total of 14 patients from two previous studies done at Kenyatta National Hospital and Mbagathi District Hospital (12, 13). For the bronchoscopy procedure patients were admitted to the Nairobi Hospital under the respective projects.

Laboratory procedures: Sputa were liquefied with dithiothreitol and diluted at a 1:1000 ratio with sterile distilled water, after which this dilution was inoculated onto blood and chocolate (Oxoid) plates. Similarly, throat swabs were inoculated onto blood and chocolate agar plates. $5 \mathrm{ml}$ venous blood or bronchoalvelar lavage for culture was obtained from patients before antibiotic treatment was commenced and cultured in $50 \mathrm{ml}$ broth media containing brain heart infusion and para-aminobenzoic acid. Broth cultures showing signs of bacterial growth were subcultured onto blood, chocolate and macConkey agar plates. Inoculated plates were incubated at $37^{\circ} \mathrm{C}$ in a $5 \% \mathrm{CO}_{2}$ atmosphere for 24 hours. On blood agar $S$. pneumoniae were differentiated from other alpha haemolytic streptococci by including an optochin (ethylhydroxycupreine) disk; S. pneumoniae is inhibited by optochin. S. pneumoniae isolates were also confirmed by the bile solubility test. $\mathrm{X}$ and $\mathrm{V}$ factors required for growth of H.influenzae were used to confirm colonies of this bacterium subcultured from chocolate agar plates.

Antimicrobial susceptibility testing: Microdilution minimum inhibitory concentrations (MICs) were determined according to the National Committee for Clinical Laboratory Standards (NCCLS) guidelines(14). The following antibiotics were used; penicillin (S. pneumoniae only), ampicillin (H. influenzae only), amoxycillin, amoxycillin/clavulanate (2:1), cefaclor, cefuroxime, cefixime, ceftriaxone, cefprozil, erythromycin, clarithromycin, azithromycin, ciprofloxacin, ofloxacin, chloramphenicol, doxycycline and cotrimoxazole. Two sets of 96-well microdilution trays were used; one for S. pneumoniae and the other for $H$. influenzae. Quality control organisms were tested for each microdilution run, and results of testing accepted only if quality control results were within limits specified by the NCCLS (14). Inoculum checks were always performed on all quality control organisms. The following quality control organisms were tested; H. influenzae (ATCC 49247), H. influenzae (ATCC 49766) and S. pneumoniae (ATCC 49619). B-lactamase testing of all $H$. influenzae isolates was performed by the nitrocefin disk method (Difco). A positive control (a ß-lactamase positive $H$. influenzae) and a negative control (such as H. influenzae, ATCC 10211) were used on each day of testing.

\section{RESULTS}

Bacterial isolates: During the two year study a total of $277 \mathrm{~S}$. pneumoniae and $58 \mathrm{H}$. influenzae were obtained from 536 adults examined for suspected communityacquired pneumonia. Of these five $S$. pneumoniae and $2 \mathrm{H}$. influenzae were isolated from blood and BAL of separate patients (from a total of 14 from whom blood and BAL had been obtained). Out of a total of 15 patients from whom sputum could not be obtained in the course of the study three $S$. pneumoniae strains were isolated from throat swabs. In addition 16 patients carried both $S$. pneumoniae and $H$. influenzae, which were isolated from sputa.

Table 1

MICs $(\mu \mathrm{g} / \mathrm{mL})$ of S. pneumoniae from community acquired pneumonia in adults, 1998-99 $(n=277)$

\begin{tabular}{lcccc}
\hline Antimicrobial Agent & Range & Mode & MIC50 & MIC90 \\
\hline Penicillin & $0.015-4$ & 0.015 & 0.03 & 0.5 \\
Amoxycillin & $0.015-4$ & 0.03 & 0.03 & 0.12 \\
Amoxycillin/clavulanate & $0.015-2$ & 0.03 & 0.03 & 0.12 \\
Cefaclor & $0.5-64$ & 0.5 & 1 & 2 \\
Cefuroxime & $0.015-8$ & 0.03 & 0.12 & 1 \\
Cefixime & $0.12-16$ & 0.25 & 0.5 & 4 \\
Ceftriaxone & $0.015-1$ & 0.015 & 0.03 & 0.25 \\
Cefprozil & $0.12-16$ & 0.12 & 0.25 & 0.5 \\
Erythromycin & $0.015-32$ & 0.06 & 0.06 & 0.06 \\
Clarithromycin & $0.015-32$ & 0.03 & 0.03 & 0.03 \\
Azithromycin & $0.03-32$ & 0.06 & 0.06 & 0.12 \\
Ciprofloxacin & $0.5-4$ & 1 & 1 & 2 \\
Ofloxacin & $1-8$ & 2 & 2 & 2 \\
Chloramphenicol & $1-16$ & 2 & 2 & 4 \\
Doxycycline & $0.06-8$ & 0.12 & 0.12 & 8 \\
Co-trimoxazole & $0.06-8$ & 4 & 1 & 8 \\
\hline
\end{tabular}

Antimicrobial susceptibility of S. pneumonia: Of the 277 S. pneumoniae strains studied there was good susceptibility to the $\beta$-lactamclass of antimicrobials tested, except penicillin (43.3\% non-susceptible), Cefaclor (27.8\% non-susceptible) and cefixime (100\% non-susceptible). The macrolides also fared well. The quinolones are more difficult to interpret, as there is no NCCLS breakpoint defined for ciprofloxacin. However, ofloxacin yielded $2.9 \%$ non susceptible strains. If a PK/PD-derived breakpoint is used for ciprofloxacin $(\leq 0.5 \mu \mathrm{g} / \mathrm{mL})$ then $88.1 \%$ strains are non-susceptible. The older agents, namely chloramphenicol, doxycycline and co-trimoxazole, were not so effective with non-susceptibility rates of $3.2 \%$, $24.2 \%$ and $50.5 \%$, respectively. Figure 1 shows the percentage susceptibility of isolates and Table 1 is a summary of MIC values for $S$. pneumoniae strains. 
Figure 1

Susceptibility patterns of S. pneumoniae isolates

What was particularly worrying was the high number of multidrug resistant strains (Table 3 ). Only $58.8 \%$ strains showed no resistance with $7.6 \%$ strains being resistant to two or more agents. The least effective antimicrobial agents were cefixime, ciprofloxacin (with PK/PD) breakpoint of $(\leq 0.5 \mu \mathrm{g} / \mathrm{mL})$, co-trimoxazole, penicillin, cefaclor and doxycycline, with nonsusceptibility rates of $100 \%, 88.1 \%, 50.5 \%, 43.3 \%, 27.8 \%$ and $24.2 \%$, respectively. The most effective agent was amoxycillin (including amoxycillin/clavulanate) with $100 \%$ efficacy.

Table 2

MICs of $H$. influenzae from community acquired pneumonia in adults, 1998-1999 $(n=58)$

\begin{tabular}{lcccc}
\hline Antimicrobial agent & Range & Mode & MIC50 & MIC90 \\
\hline Ampicillin & $0.12-16$ & 0.12 & 0.12 & 0.5 \\
Amoxycillin & $0.0125-16$ & 0.5 & 0.5 & 1 \\
Amoxycillin/clavulanate & $0.12-1$ & 0.25 & 0.5 & 0.5 \\
Cefaclor & $0.5-8$ & 2 & 2 & 4 \\
Cefuroxime & $0.25-1$ & 0.5 & 0.5 & 1 \\
Cefixime & $0.008-0.12$ & 0.03 & 0.03 & 0.06 \\
Ceftriaxone & $0.004-0.008$ & 0.004 & 0.004 & 0.008 \\
Cefprozil & $0.5-8$ & 1 & 2 & 2 \\
Erythromycin & $1-8$ & 4 & 4 & 8 \\
Clarithromycin & $2-16$ & 8 & 8 & 8 \\
Azithromycin & $0.12-2$ & 0.5 & 0.5 & 2 \\
Ciprofloxacin & $0.008-0.03$ & 0.015 & 0.015 & 0.015 \\
Ofloxacin & $0.015-0.25$ & 0.03 & 0.03 & 0.03 \\
Chloramphenicol & $0.25-8$ & 0.5 & 0.5 & 0.5 \\
Doxycycline & $0.25-2$ & 0.5 & 0.5 & 1 \\
Co-trimoxazole & $0.03-4$ & 4 & 4 & 4 \\
\hline
\end{tabular}

Antimicrobial susceptibility of $H$. influenzae: Of the $58 \mathrm{H}$. influenzae strains examined, only $8.6 \%$ produced $\mathrm{B}$ lactamases, and these accounted for all the ampicillinresistant strains $(8.6 \%)$. The other B-lactams all showed excellent efficacy with $>90 \%$ susceptibility. The macrolides also demonstrated good efficacy (clarithromycin and azithromycin $>90 \%$ susceptibility). However, no NCCLS breakpoint has been set for erythromycin, but if a PK/PD derived breakpoint of $\leq 0.25$ is applied to the data $100 \%$ strains are resistant to this agent. Chloramphenicol and doxycycline show good efficacy of $94.8 \%$ and $100 \%$ susceptibility, respectively. Cotrimoxazole has only $44.8 \%$ susceptibility. Figure 2 shows the percentage susceptibility of isolates and Table 2 is a summary of MIC values for $H$. influenzae.

Figure 2

Susceptibility patterns for $\mathrm{H}$. influenzae isolates

Multi-resistant strains were also prevalent amongst the haemophilus isolates (Table 3). All strains were nonsusceptible to erythromycin (using a PKPD breakpoint of $\leq 0.25$ ) and co-trimoxazole gave a resistance rate of $55.2 \%$. All other antimicrobial agents tested gave nonsusceptibility rates of $<10 \%$.

Table 3

Prevalence of multi-resistant strains 1998-99: percent of resistance to $n$ antimicrobial agents

\begin{tabular}{lcc}
\hline & S. pneumoniae & H. influenzae \\
\hline Total strains & 277 & 58 \\
Resistant to 0 agents & $58.8 \%$ & $46.6 \%$ \\
Resistant to 1 agents & $33.6 \%$ & $46.6 \%$ \\
Resistant to 2 agents & $6.5 \%$ & $3.4 \%$ \\
Resistant to 3 agents & $1.1 \%$ & $3.4 \%$ \\
Resistant to 4 agents & $0 \%$ & $0 \%$ \\
Resistant to 5 agents & $0 \%$ & $0 \%$ \\
Resistant to $\geq 6$ agents & $0 \%$ & $0 \%$ \\
\hline
\end{tabular}

\section{DISCUSSION}

In most countries, Streptococcus pneumoniae and Haemophilus influenzae are the major causes of community-acquired pneumonia $(5,9,15)$. Furthermore, the prevalence of multidrug resistance (resistant to two or more antimicrobial agents) among $S$. pneumoniae has been on the increase worldwide (6-9) and our study observed a $7.6 \%$ prevalence of multidrug resistance. In addition, previous studies from Kenya found that 
pneumococcal isolates did not show full resistance to penicillin although the prevalence of intermediate penicillin resistance has increased over the last two decades $(3,16)$. However, for treatment of acute pneumonia high dose penicillin (150,000- 200,000 units/kg/day) is still regarded as effective but the high number of non-susceptible isolates in this study may result in high clinical failure rates (3).

Only $1.8 \%$ of $S$. pneumoniae were resistant to penicillin while resistance to erythromycin and chloramphenicol was $<5 \%$, a much lower prevalence compared to figures from the industrialised countries (5). Of great concern from our study, however, was the high level of resistance to cotrimoxazole $(38.3 \%)$ and doxycycline (14.8\%). Similarly, Scott et al (3) observed prevalence of $54 \%$ and $32 \%$ to cotrimoxazole and tetracycline, respectively. These two drugs are among the most commonly available in most public hospitals for treatment of acute respiratory tract infections and other bacterial infections, and the high level of resistance would render them less effective for treatment of these infections. As in other studies from African countries $(8,10,17)$ pneumococcal isolates from our study were susceptible to semi-synthetic penicillins and extended spectrum cephalosporins, but these drugs may be unavailable or are too expensive to be afforded by most patients who seek care at public hospitals in Kenya.

Resistance to ß-lactams in Haemophilus influenzae is due to production of TEM-1 B-lactamases (95\%) and ROB-1 enzymes (5\%), both enzymes are plasmidmediated. In the USA since 1983, the prevalence of $B-$ lactamase producing $H$. influenzae rose from $14.5 \%$ to $37.5 \%$ in 1997, providing resistance to penicillin and first generation cephalosporins (10). In our study only $8.6 \%$ of H. influenzae produced B-lactamase although these were not characterised further. However, resistance to cotrimoxazole $(51.7 \%)$, which is commonly used in outpatient treatment of acute respiratory tract infections, is worrying. Also of importance was the high prevalence of resistance to erythromycin (100\%), which has been used in Kenya as second line treatment for acute respiratory tract infections. These trends may reflect overuse and sometimes misuse of these antimicrobials.

In conclusion our study observed that resistance to many of the first line antimicrobials used in Kenya (e.g. penicillin, cotrimoxazole, doxycycline, and erythromycin) for the treatment of community-acquired pneumonia is high, and that there is a trend to increasing resistance in the common aetiological agents. The World Health Organisation guidelines for treatment of outpatient acute respiratory tract infection recommend either cotrimoxazole or amoxycillin. Clearly for Kenya, cotrimoxazole would not be effective. However, this study does support the use of B-lactams. Furthermore, it may be worth considering a $\beta$-lactam/ B-lactamase inhibitor combination or a ß-lactamase-stable cephalosporin due to the incidence of $\beta$-lactamase-producing Haemophilus influenzae. As it is generally well established that the emergence of resistance among bacteria is an inevitable event following the use of antibiotics, the introduction of new and more potent antimicrobial agents will give an edge in this war but equally important will be efforts directed at delaying or minimising the likelihood for the emergence and subsequent dissemination of resistance by instituting prudent and appropriate use of available antimicrobials.

\section{DECLARATION OF INTEREST}

William Mwatu, a pharmacist and J.J. Daniels, a Clinical Microbiologist are employees of Glaxo-SmithKline. Both participated in the write-up of proposal for the study that was part of a larger multinational "Alexander Project" on surveillance of antibiotic resistance and in final report writing, hence qualify as authors. However, none of them was involved in the laboratory testing of antimicrobial susceptibility of isolates reported in this paper.

\section{ACKNOWLEDGEMENTS}

To the Director, Kenya Medical Research Institute for permission to publish this work. This study was supported by a grant from GlaxoSmithKline.

\section{REFERENCES}

1. Koulla-Shiro, S., Kuaban, C. and Belec, L. Acute communityacquired bacterial pneumonia in, human immunodeficiency virus (HIV) infected and non-HIVinfected adult patients in Cameroon: aetiology and outcome, Tubercle. Lung .Dis. 1996; 77:47-51.

2. Mwachari, C.W., Cohen, C.R., Meier, A.S., Nganga, L.W., Kimari, J.N. and Odhiambo, J.A. Respiratory tract infection in HIV-1-infected adults in Nairobi, Kenya. Evaluation of risk factors and the World Health Organization treatment algorithm. J. AIDS . 2001; 27:365-371.

3. Scott, J.A.G., Hall, A.J., Muyodi, C. et al. Aetiology, outcome and risk factors for mortality among adults with acute pneumonia in Kenya. Lancet. 2000; 355:1225-1230.

4. Hawkes, C.A. Antibiotic resistance: a clinician's Perspective. Mil Med, 2000; 165(Suppl 2):43-45.

5. Lister, P.D. Emerging resistance problems among respiratory tract pathogens Amer. J. Manag. Care2000; 6(8 Suppl):S409S418.

6. Johnson, A.P., Warner, M., George, R.C. and Livermore, D.M. Activity of moxifloxacin against clinical isolates of Streptococcus pneumoniae from England and Wales. J. Antimicrob. Chemother. 2001; 47:411-415.

7. Soewignjo, S., Gessner, B.D., Sutanto, A. et al. Streptococcus pneumoniae nasopharyngeal carriage prevalence, serotype distribution, and resistance patterns among children on Lombok Island, Indonesia.Clin. Infect. Dis. 2001;32:10391043.

8. Huebner, R.E., Wasas, A.D. and Klugman, K.P. Trends in antimicrobial resistance and serotype distribution of blood and cerebrospinal fluid isolates of Streptococcus pneumoniae in South Africa, 1991-1998. Int. J. Infect. Dis. 2000; 4:214218.

9. Critchley, L.A., Thornsberry, C., Piazza, G., Yamakita, J., Barth, A., Sader, H.S. et al. Antimicrobial susceptibility of Streptococcus pneumoniae, Haemophilus influenzae and Moraxella catarrhalis collected from five centers in Brazil, 1997-98. Clin. Microbiol. Infect. 2000; 6:178-184. 
10. Benbachir, M., Benredjeb, S., Boye, C.S. et al. Two-year surveillance of antibiotic resistance in Streptococcus pneumoniae in four African cities. Antimicrob. Agents Chemother. 2001; 45:627-629.

11. Karlowsky, J.A., Verma, G., Zhanel, G.G. and Hoban, D.J. Presence of ROB-1 beta-lactamase correlates with cefaclor resistance among recent isolates of Haemophilus influenzae. J. Antimicrob. Chemother. 2000; 45:871-875.

12. Paul, J., Bates, J., Kimari, J. and Gilks, C. Serotypes and antibiotic susceptibilities of Streptococcus pneumoniae in Nairobi, Kenya, J. infect. 1996; 32:139-142.

13. Arthur, G., Nduba, V.N., Kariuki, S.M., Kimari, J., Bhatt, S.M. and Gilks, C.F Trends in bloodstream infection among human immunodeficiency virus-infected adults admitted to a hospital in Nairobi, Kenya, during the last decade. Clin. Infect. Dis. 2001; 33:248-256.

14. National Committee for Clinical Laboratory Standards. Method for dilution antimicrobial susceptibility tests for bacteria that grow aerobically. Approved standard M7-A5. National Committee for Clinical Laboratory Standards, Wayne, Pa, 2000.

15. Levine, O.S., Liu, G., Garman, R.L., Dowell, S.F., Yu, S. and Yang, Y. Haemophilus influenzae type b and Streptococcus pneumoniae as causes of pneumonia in Beijing, China.Emerg. Infect. Dis. 2000; 6:165-170.

16. Scott, J.A., Hall, A.L., Hannington, A., Edwards, R., Mwarumba, S., Lowe, B. et al. Serotype distribution and prevalence of resistance to benzylpenicillin in three representative populations of Streptococcus pneumoniae isolates from the coast of Kenya. Clin. Infect. Dis. 1998; 27:1442-1450.

17. Klugman, K.P. The clinical relevance of in-vitro resistance to penicillin, ampicillin, amoxycillin, and alternative agents, for the treatment of community acquired pneumonia caused by Streptococcus pneumoniae, Haemophilus influenzae and Moraxella catarrhalis. J. Antimicrob Chemother. 1996; 38:133-140.

\section{TREATMENT OF SNAKE BITE SOUTH AFRICAN VACCINE PRODUCERS (PTY) LTD}

"BUYING FROM AFRICA FOR AFRICA"

AS AWHOLLY OWNED SUBSIDIARY OF THE NATIONAL HEALTH LABORATORY SERVICES (PREVIOUSLY S.A.I.M.R) S.A.V.P (PTY) LTD IS COMMITTED TO CONTINUE ITS PRODUCTION OF STRATEGIC ANTIVENOM PRODUCTS, USED SUCCESSFULLY FOR THE TREATMENT OF ENVENOMATION BY POISONOUS AFRICAN SNAKES. WE HAVE BEEN OPERATIONAL SINCE 1928.

\section{OUR PRODUCTS INCLUDE:}

1) POLYVALENT SNAKE ANTIVE NOM T517(ACT 101/1965)

EFFECTIVE AGAINST THE FOLLOWING SPECIES:

Bitis arietans (Puff adder)

Bitis gabonica (gaboon adder)

Hemachatus Haemachatus (rinkhals)

Dendroaspis angusticeps (green mamba)

Dendroaspis polylepsis (black mamba)

Dendroaspis jamesoni (J amesons mamba)

Naja nivea (cape cobra)

Naje melanoleuca (forest cobra)

Naje haje annulifera (Egyptian cobra)

Naje mossambica (Mozambican spitting cobra)

2) SPECIFIC BOOMSLANG MONOVALENT ANTIVENOM T 515 (ACT 101/ 1965)

3) MONOVALENT ANTIVENOM AGAINST THE SAW SCALED VIPER (E CHIS CARINATUS/OCE LLATUS) T516(ACT101/1965)

FOR FURTHER INFORMATION PLEASE CONTACT US ON +27 (11)-386 6000 OR VIEW OUR WEBSITE AT www.savp.co.za 